\title{
COX-2 expression correlation with invasion in human breast carcinomas and in cell line MDA-MB-231 in vitro
}

\author{
Gang-Ping Wang1, Ying Chen², Bai-Li An³, Ying Zhao4, Bei-Bei Sui4, Peng-Xiang Min4, \\ Yun Zhao5, Yun-Ai Liang' and Yi-Nuo Zhao' \\ ${ }^{1}$ Department of Pathology, Rizhao People's Hospital, Affiliated Jining Medical University, Rizhao, Shandong \\ 276 826, China; ' 2 Department of Medical Laboratory, Rizhao People's Hospital, Rizhao, Shandong 276 826, China; \\ ${ }^{3}$ Department of general surgery, Rizhao People's Hospital, Rizhao, Shandong 276 826, China; ${ }^{4}$ Biological Science \\ College, Jining Medical University, Rizhao, Shandong 276 826, China; ${ }^{5}$ Pathology Department of Clinical \\ Laboratory Center, Tai'an Cancer Prevention and Control Institute, Tai'an, Shandong 271 000, China.
}

\begin{tabular}{|lr|}
\hline Article Info & \\
\hline Received: & 11 November 2015 \\
Accepted: & 2 February 2016 \\
Available Online: & 3 March 2016 \\
DOI: $10.3329 /$ bjp.v11iS1.25639
\end{tabular}

Cite this article:

Wang GP, Chen Y, An BL, Zhao Y, Sui BB, Min PX, Zhao Y, Liang YA, Zhao YN. COX-2 expression correlation with invasion in human breast carcinomas and in cell line MDA-MB231 in vitro. Bangladesh J Pharmacol. 2016; 11: S43-S54.

\begin{abstract}
COX-2 expression by means of immunohistochemistry in 120 cases breast invasive ductal carcinomas and 60 cases benign lesions were compared with clinicopathological features and prognostic molecular markers. COX-2 role of invasiveness and chemotaxis in breast cancer cell line MDA-MB-231 by using small RNA interference (siRNA) plasmids to disrupt COX-2 expression in vitro were investigated. The results show that COX-2 immune positivity and percentage of positive cells in breast carcinomas were higher than those in benign lesions and positivity correlated significantly with HIF-1a, VEGF, the grading, metastasis and vascular invasion of carcinoma $(p<0.05)$. This study suggests that COX-2 overexpression correlates with poor clinicopathological parameters in breast cancers, and the reduction of COX-2 expression can obviously inhibit the invasion and chemotaxis of cancer cell line MDA-MB231 using RNA interference. The findings of the present study suggest that COX-2 overexpression in breast cancer may be considered as a negative prognostic marker.
\end{abstract}

\section{Introduction}

Breast cancer is one of the commonly-encountered malignant tumors (Lakhani et al., 2012; Thorat et al., 2013), and is the second leading cancer causing death in women all over the world (Elston et al., 1991; Yi et al., 2014; Wang et al., 2014). It is mainly a multifactorial disease, occurring as a result of the combined effects of factors (Khan et al., 2015; Aggarwal et al., 2014). During the past decades, there has been an increasing interest in their relationship between the cyclooxygenase type-2 (COX-2) overexpress in tumor and their relationship

This paper was presented in the 3rd International Conference on Biomedicine and Pharmaceutics in Zhuhai, China, on December 11-13, 2015. with the biological behavior (Miglietta et al.,2010; Olivan, et al., 2015). Several studies have suggested that a possible role for COX-2 in carcinogenesis, tumor progression, involved in proliferation, apoptosis, angiogenesis and invasion (Miglietta et al., 2010 ). However, other studies reported no significant correlations between COX-2 expression and histopathological parameters or DFS (Ranger et al., 2004; Kelly et al., 2003). T

The role for COX-2 on breast cancer is not fully clear, and studies have shown that the proliferation and metastasis of breast cancer cells is a very complex, multistep process, affected by various factors including genetic and so on (Miglietta et al., 2010; Yu et al., 2014). The aim of this study was to investigate COX-2 expression in human breast cancers tissues and its 
possible association with clinicopathological features and prognostic molecular markers such as human epidermal growth factor receptor type 2 (HER2/neu), ER, PR, Ki-67 proliferation index, HIF-1a and VEGF, etc., and investigated by reducing COX-2 using RNA interference highly malignant breast cancer cell line MDA-MB-231 transfected with small RNA interference (siRNA) plasmids to disrupt COX-2 expression.

\section{Materials and Methods \\ Patients and clinicopathological analyses}

One hundred twenty-eight patients diagnosed breast invasive cancer were collected during excision surgery at Rizhao People's Hospital from June 2000 to June 2013. The patients ranged in age from 21 to 78 years, and the mean age was $47.5 \pm 5.3$ years. The patients' body weight ranged from 42 to $79 \mathrm{~kg}$, and the mean body weight was $55.8 \pm 4.7 \mathrm{~kg}$. No patient was treated with radiotherapy, chemotherapy, or endocrine therapy before surgery. Sixty cases of benign lesions were selected as a control group. In the benign breast lesion group, the patient ages ranged from 22 to 74 years, and the mean age was $46.2 \pm 5.1$ years. The patients' body weight ranged from 42 to $73 \mathrm{~kg}$, and the mean body weight was $55.3 \pm 6.7 \mathrm{~kg}$. No significant difference was noted regarding general data such as gender, age, and body weight, indicating good comparability between the observation group and benign breast lesion group $(\mathrm{p}>0.05)$. For analyses, we considered the age at diagnosis, menopausal status, tumor size, histological grading, lymph node involvement, stage, status of ER, PR, HER2/neu, HIF-1a and VEGF expression, and the $\mathrm{Ki}-67$ indexin relation to the COX-2 expression. Data concerning ER, PR, HER2/neu, HIF-1a and VEGF status, Ki-67 proliferation index, and histological grade were recorded. Patients assessed for the expression of ER, PR, HER2/neu, survivin, HIF-1a and VEGF were grouped as positive or negative. Regarding the $\mathrm{Ki}-67$ proliferation index, patients were divided into two groups: Those with a Ki-67 proliferation index between $1 \%$ and $14 \%$ and those with proliferation index $>14 \%$. Concerning the histological grade, patients were classified into two groups: Grade I or II, Grade III.

The pathological diagnosis was verified by histological methods independently by two pathologists, and pathological categorization was determined according to the current World Health Organization classification system, and the pathological diagnosis was verified by histological methods independently by two pathologists, and the pathologists were blinded to the subject's clinical history and the results of the immunohistochemistry staining assay. The pathological reading was determined for each biopsy slide with an overall pathological diagnosis determined for each subject. The tumor grade was determined according to the modified Bloom-Richardson score (Thorat et al., 2013). The grade was obtained by summing the scores for tubule formation, nuclear pleomorphism, and mitotic count, which were scaled as 1,2 , or 3 . The final scores ranged between 3 and 9 and were then divided into three grades (I-III). The final grading scores were as follows: Sum of points, 3-5, final grade I; 6-7, II; and 8-9, III. The pathologists were blinded to the subject's clinical history and the results of the immunohistochemistry staining assay. According to the International Union against Cancer (UICC) TNM classification of solid malignant tumors standard (7th ed) and American Joint Committee on Cancer (AJCC) Cancer Staging Manual (7th ed) were used to evaluate the therapeutic effect.

\section{Immunohistochemistry}

Breast tissue samples were fixed in $10 \%$ neutral buffered formalin and embedded in paraffin at $4^{\circ} \mathrm{C}$ for 24 hours. Tissue sections at $5 \mu \mathrm{m}$ thickness were deparaffinized and rehydrated using standard procedures. The specimens were examined under a binocular-dissecting microscope. Immunoreactions were processed using the Ultra Sensitive ${ }^{T M}$ S-P Kit (Maixin-Bio, China) according to the manufacturer's instructions to detect differences in tumor tissue COX-2, HIF-1a, VEGF and CD105 expression, and signals were visualized using the DAB substrate, which stains the target protein yellow. The pathological specimens were reviewed independently by two pathologists and the pathologists were blinded to the subject's clinical history, and the results of the immunohistochemistry staining assay.

For COX-2, the immunoreactive score (IRS) was obtained by multiplying the percentage of positive cells and the staining intensity. Immunostaining was recorded according to stain intensity (intensity score) and percentage of cancerous cells that stained positively (quantity score). Briefly, a proportion score was assigned that represented the estimated proportion of positive tumor cells on the entire slide. For each histological section, the percentage of positive cells was scored as $0(<5 \%), 1$ $(6 \%-25 \%), 2(26 \%-50 \%), 3(51 \%-75 \%)$, and $4(>75 \%)$, and the staining intensity was scored as 0 (negative), 1 (weak), 2 (moderate), and 3 (strong). Immunohistochemical results with an IRS of 0 were considered negative, an IRS of 1-4 was considered weakly positive, an IRS of 5-8 was considered moderately positive, and an IRS of 9-12 was considered strongly positive. The negative controls were used. The primary antibody was replaced with PBS, containing $0.1 \%$ bovine serum albumin at the same concentration as the primary antibody. The positive controls were tissues known to express the antigen being studied.

The immunoreactivity of positivity for VEGF was indicated by cytoplasmic staining, and HIF-1a was expressed as the percentage of cancer cells showing 
nuclear reactivity. For VEGF and HIF-1a, cells were classified according to the positive rate and color intensity as follows: Negative, number of positive cells $<25 \%$; positive, brown particles, number of positive cells $\geq 25 \%$.

For ER, PR, and Ki-67 expression, the percentage of cancer cells showing nuclear reactivity was recorded after inspection of all of optical fields at $\times 200$, and the mean value was used to score each case. Tumors showing expression in $>1 \%$ of cancer cells were considered positive. ER and/or PR positivity was considered combined ER and PR positivity, and combined ER and PR negativity was considered both ER and PR negativity. For the Ki-67 proliferation index, patients were divided into two groups: Those with a value between $1 \%$ and $14 \%$ and those with a value $>14 \%$.

Histologically recognizable vessels within tissue sections served as internal controls for CD105 immunostaining. The MVD was evaluated under light microscopy according to the procedure described (Kopczyńska and Makarewicz, 2012). Briefly, after scanning the sections at low magnifications, five tumor areas with the greatest number of distinctly highlighted micovessels were selected. The number of vessels was counted in the highlighted micovessels at high magnifications $(400 \times)$, and the average counts of the fields were recorded. Each brown-stained endothelial cell or endothelial cell cluster, which was clearly separate from the adjacent micovessels, tumor cells and connective tissue elements was considered a single, countable microvessel. Areas of fibrosis, necrosis and inflammation, and vessels with muscular walls were not counted.

In addition, regarding the cell membrane reactivity of HER2/neu, oncoprotein expression was evaluated following a similar approach, and the mean value was used to score each case. Tumors expressing HER2/neu in $>10 \%$ of the cancer cells were considered to show positive expression.

In vitro studies by reducing COX-2 by using RNA interference in cells line MDA-MB-231

In vitro studies, to investigate the role of COX-2 in invasiveness and chemotaxis of the highly malignant breast cancer cell line MDA-MB-231 RNA interference was used. The MDA-MB-231 cells were cultured. The small interfering RNA expression vectors targeting COX-2 was constructed. MDA-MB-231 cells were transfected with small RNA interference plasmids to disrupt COX-2 expression. COX-2 mRNA levels were detected using RT-PCR. Chemotaxis assay and scratch assay were examined to detect the migration and chemotaxis ability of MDA-MB-231 cells. The adhering ability of MDA-MB-231cells was examined using adhesion assay. The in vitro invasion ability of MDA-
MB-231 cells was examined using matrigel invasion assay.

\section{Statistical analysis}

SPSS version 17.0 statistical software (SPSS Inc., USA) was used to analyze the data. Enumeration data with chi-squared $\left(\chi^{2}\right)$ test. The relationship of this dichotomous variable to other clinicopathological correlates was established using $\chi^{2}$ or Fisher's exact tests, as appropriate. The MVD results were expressed as the mean and standard deviation (SD). Because the results which were measured in tumor tissues, was not Gaussian, the nonparametric Mann-Whitney U-test was used to determine differences between the benign and malignant groups. Kaplan-Meier overall survival curves were constructed to demonstrate the survival differences between the COX-2 positive and COX-2 negative patients. All other statistical tests were performed using Graphpad Prism 5.0 (Graphpad Software Inc., USA). A $\mathrm{p}$ value less than 0.05 was deemed statistically significant.

\section{Results \\ Clinical features and follow-up date}

A summary of the results concerning major clinicopathological parameters, including pathological tumor stage, nodal status, hormone receptor status, tumor size, grading and vascular space involvement is provided in Table I.

COX-2 Immunohistochemical express and relationship with clinicopathological factors

\section{COX-2 express in different groups}

COX-2 immune positivity in tumor cells was appreciated by immunohistochemistry as a diffuse, granular cytoplasmic staining in breast lobules. The expressions of COX-2 in observation and control groups were shown in Figure 1. Benign tissues, COX-2 was weekly expressed only $15.4 \% \quad(10 / 60)$. Immunohistochemical analysis revealed some degree of positivity in $78.9 \%(101 / 128)$ of the tumors examined, and the positive rates of COX-2 in breast carcinomas were higher than in benign tissues $(\mathrm{p}<0.05)$. The degree of COX-2 expression differed between Grade III and Grade I or II, the normal tissue away from the tumor did not stain with COX-2 (results not shown).

\section{Correlations between COX-2 positivity and clinico- pathological factors in breast cancers}

Relationship between COX-2 and clinicopathological parameters of breast cancer was shown in Table II. Statistical analysis revealed significant relationships between COX-2 expression and clinical parameters such as histoligical grading, extent of primary tumor, vascular invasion, lymph node metastasis, distant metastasis 


\begin{tabular}{|c|c|c|}
\hline \multicolumn{3}{|c|}{ Table I } \\
\hline \multicolumn{3}{|c|}{ Clinicopathological data of patients } \\
\hline Biological parameters & $\mathrm{n}$ & $\%$ \\
\hline \multicolumn{3}{|l|}{ Age at diagnosis } \\
\hline$\leq 50 \mathrm{yr}$ & 60 & 46.9 \\
\hline$>50 \mathrm{yr}$ & 68 & 53.1 \\
\hline \multicolumn{3}{|l|}{ Tumor size } \\
\hline$\leq 5 \mathrm{~cm}$ & 109 & 85.2 \\
\hline$>5 \mathrm{~cm}$ & 19 & 14.8 \\
\hline \multicolumn{3}{|l|}{ Histoligical grade } \\
\hline I or II & 96 & 75.0 \\
\hline III & 32 & 25.0 \\
\hline \multicolumn{3}{|l|}{ Vascular invosion } \\
\hline Present & 52 & 40.6 \\
\hline Absent & 76 & 59.4 \\
\hline \multicolumn{3}{|l|}{ Lymph node metastasis } \\
\hline Present & 83 & 64.8 \\
\hline Absent & 45 & 35.2 \\
\hline \multicolumn{3}{|l|}{ Distant metastasis } \\
\hline Present & 37 & 28.9 \\
\hline Absent & 91 & 71.1 \\
\hline \multicolumn{3}{|l|}{ Recurrence } \\
\hline Positive & 22 & 17.2 \\
\hline Negative & 106 & 82.8 \\
\hline \multicolumn{3}{|l|}{ Vascular invosion } \\
\hline Present & 52 & 40.6 \\
\hline Absent & 76 & 59.4 \\
\hline \multicolumn{3}{|l|}{ ER status } \\
\hline Positive & 108 & 84.4 \\
\hline Negative & 20 & 15.6 \\
\hline \multicolumn{3}{|l|}{ PR status } \\
\hline Positive & 110 & 85.9 \\
\hline Negative & 18 & 14.1 \\
\hline \multicolumn{3}{|l|}{ HER2 statuse } \\
\hline Positive & 33 & 25.8 \\
\hline Negative & 95 & 74.2 \\
\hline \multicolumn{3}{|l|}{ COX-2 } \\
\hline Positive & 101 & 78.9 \\
\hline Negative & 27 & 21.1 \\
\hline \multicolumn{3}{|l|}{ HIF-1a statuse } \\
\hline Positive & 88 & 68.8 \\
\hline Negative & 40 & 31.2 \\
\hline \multicolumn{3}{|l|}{ Survivin statuse } \\
\hline Positive & 86 & 67.2 \\
\hline Negative & 42 & 32.8 \\
\hline \multicolumn{3}{|l|}{ VEGF statuse } \\
\hline Positive & 88 & 68.8 \\
\hline Negative & 40 & 31.2 \\
\hline
\end{tabular}

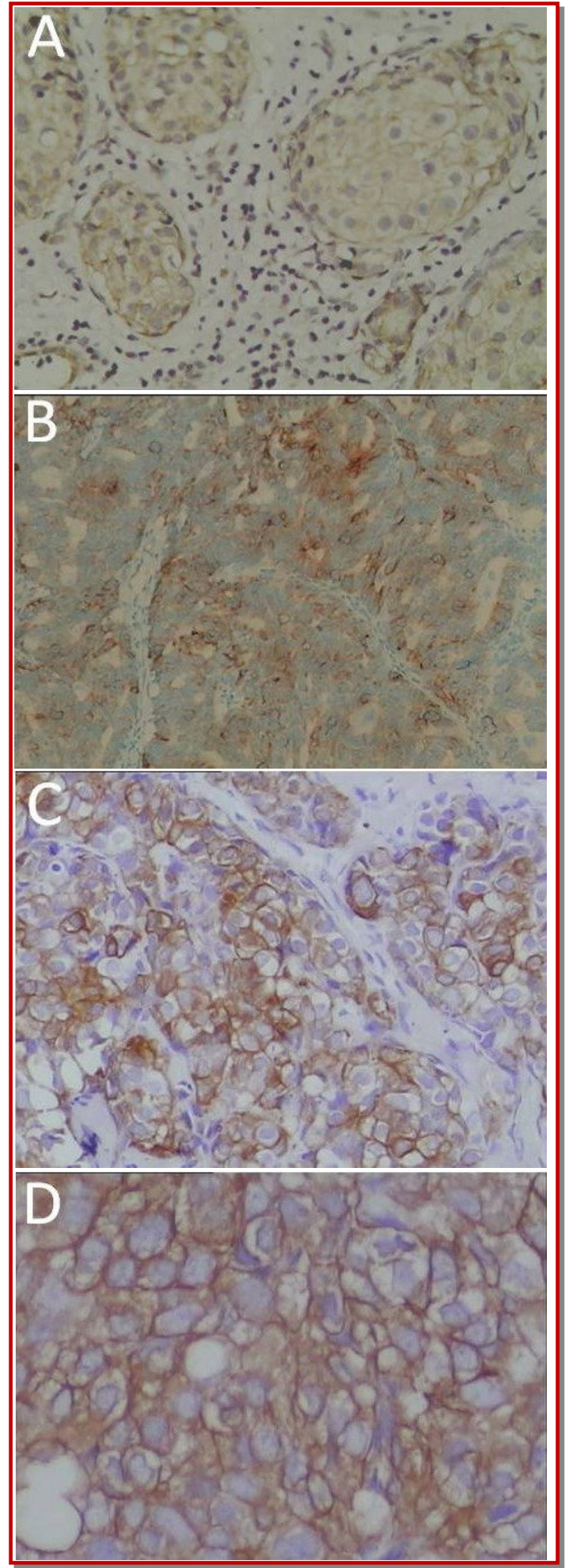

Figure 1: COX-2 immunohistochemical staining in tissue sections of breast carcinoma 


\section{Table II}

COX-2 express relation with biological parameters in breast cancer and molecular markers expresses

\begin{tabular}{|c|c|c|c|c|}
\hline Biological parameters & $\mathrm{n}$ & $\begin{array}{c}\text { COX-2 positive } \\
\text { (n) }\end{array}$ & $x^{2}$ & $\mathrm{p}$ value \\
\hline \multicolumn{5}{|l|}{ Age at diagnosis } \\
\hline$\leq 50 \mathrm{yr}$ & 60 & 48 & 0.081 & $>0.05$ \\
\hline$>50 \mathrm{yr}$ & 68 & 53 & & \\
\hline \multicolumn{5}{|l|}{ Tumor size } \\
\hline$\leq 5 \mathrm{~cm}$ & 109 & 84 & 1.497 & $>0.05$ \\
\hline$>5 \mathrm{~cm}$ & 19 & 17 & & \\
\hline \multicolumn{5}{|l|}{ Histoligical grade } \\
\hline I or II & 96 & 70 & 8.277 & $<0.01$ \\
\hline III & 32 & 31 & & \\
\hline \multicolumn{5}{|l|}{ Lymph node metastasis } \\
\hline Present & 83 & 77 & 26.403 & $<0.01$ \\
\hline Absent & 45 & 24 & & \\
\hline \multicolumn{5}{|l|}{ Distant metastasis } \\
\hline Present & 37 & 35 & 7.696 & $<0.01$ \\
\hline Absent & 91 & 66 & & \\
\hline \multicolumn{5}{|l|}{ Vascular invosion } \\
\hline Present & 52 & 42 & 4.366 & $<0.05$ \\
\hline Absent & 76 & 59 & & \\
\hline ER statuse & & 101 & & \\
\hline Positive & 108 & 83 & 1.05 & $>0.05$ \\
\hline Negative & 20 & 18 & & \\
\hline \multicolumn{5}{|l|}{ PR statuse } \\
\hline Positive & 110 & 87 & 3.42 & $>0.05$ \\
\hline Negative & 18 & 14 & & \\
\hline \multicolumn{5}{|l|}{ HER2 statuse } \\
\hline Positive & 33 & 22 & 3.07 & $>0.05$ \\
\hline Negative & 95 & 79 & & \\
\hline \multicolumn{5}{|l|}{ HIF statuse } \\
\hline Positive & 88 & 74 & 5.412 & $<0.01$ \\
\hline Negative & 40 & 27 & & \\
\hline \multicolumn{5}{|l|}{ VEGF statuse } \\
\hline Positive & 88 & 78 & 9.441 & $<0.01$ \\
\hline Negative & 40 & 23 & & \\
\hline
\end{tabular}

and recurrence, respectively, $\mathrm{p}<0.05$. By contrast, no statistically significant relationships were detected between COX-2 and the other biological parameters examined such as age at diagnosis, size of carcinoma $(\mathrm{p}>0.05)$.

\section{Correlation between COX-2 and molecular markers}

The correlation between COX-2 and molecular markers expresses in breast carcinoma was shown in Table II. COX-2 immune positivity correlated significantly with HIF-1a, VEGF, the grading, lymph node metastasis, distant metastasis and vascular invasion of carcinoma $(\mathrm{p}<0.05$, respectively), but not with biological parameters such as age, size of tumor, ER, PR and HER 2 $(p>0.05)$.

\section{COX-2 and MVD assessment in breast cancer patients}

The tissues of breast cancer and benign leisions were immunohistochemically stained for CD105 and MVD was assessed based on the number of CD105-positive vessels. CD105 positivity was indicated by membrane staining. Immunohistochemical results in observation and control groups were listed and shown in Figure 2A. The MVD had statistical significance in invasive breast cancer $(31.7 \pm 8.6)$ and benign breast leisions $(10.0 \pm 4.0)$ groups $(\mathrm{t}=22.17, \mathrm{p}<0.01)$. MVD in the peripheral area adjacent to the lesion was significantly higher than those central area within the lesion in every group 


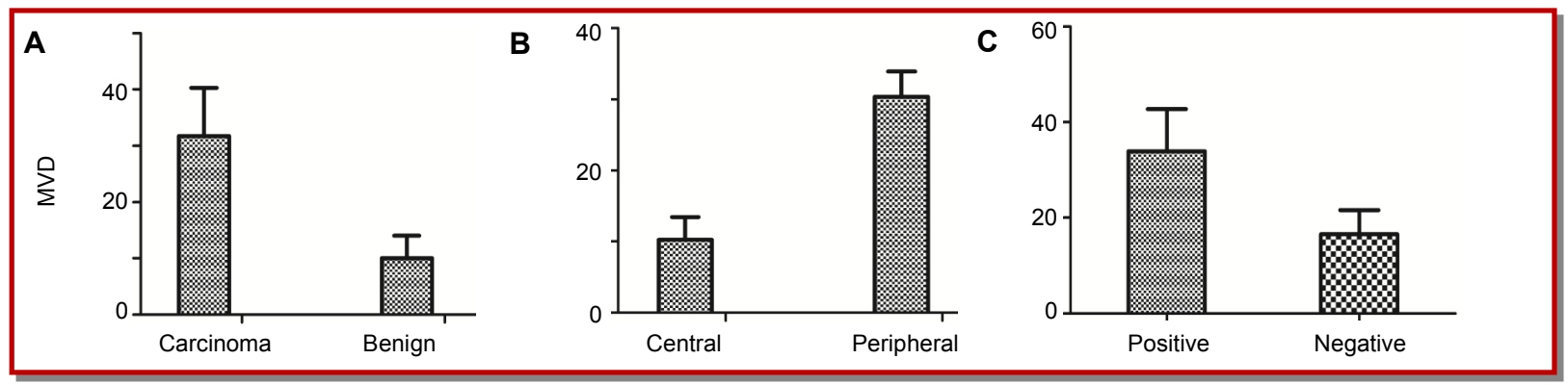

Figure 2: The MVD comparison in different groups. $\mathrm{p}<0.01$ comparison between benign and cancer (A), comparison between central and peripheral area in cancer (B), and comparison between COX-2 positive and COX-2 negative in breast cancer

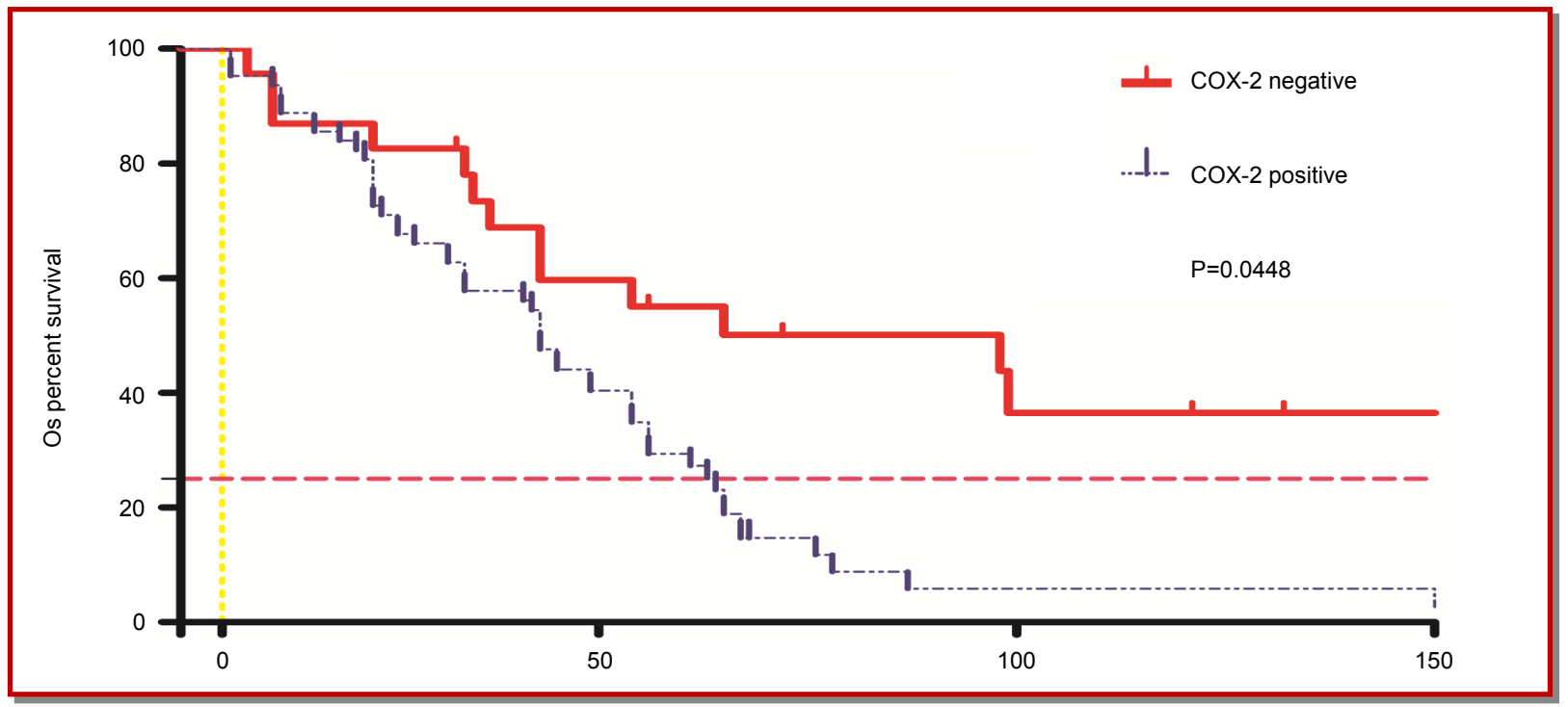

Figure 3: Kaplan-Meier overall survival curves of the patient population under study. $\mathrm{p}<0.05$ comparison between COX-2 positive and COX-2 negative in breast cancer

$(\mathrm{p}<0.01)$, shown in Figure 2B. MVD in COX-2 positive was significantly higher than those in negative in breast cancer patients shown in Figure 2C.

\section{Survival analysis}

Patients were monitored for survival from 2000 to 2014 through telephone communication and periodic returns to the rizhao people's hospital. Follow-up information was available for 86 of the patients. Clinical and pathological information documented at the time of surgery included the clinical stage of the cancer and grade and histology of the tumor. The menopausal status was also documented, and the response to chemotherapy was monitored according to the clinicopathological variables documented at the time of surgical excision and the outcome (progression-free survival (PFS), overall survival (OS) monitored over a median interval of 62 months. All of the patients were treated with either breast-conserving surgery $(n=21)$ or modified radical mastectomy $(\mathrm{n}=65)$, including axillary lymph node dissection (at least 15 nodes resected). Clinical and pathological information documented at the time of surgery included clinical stage of the cancer, grade and histology of the tumor, and amount of the remaining tumor. Patients were monitored for survival and disease progression (no apparent progression or progression) for a median duration of 62 months (range, 3-78 months). Follow-up information was available for 86 of the patients. Seventeen (19.8\%) of these patients relapsed and nine $(10.5 \%)$ died during the course of the follow-up period. Efficacy assessments were performed at 6-week intervals. Progressive disease and stable disease were assessed after the start of adjuvant treatment, and treatment response and disease progression were investigated according to the modified Response Evaluation Criteria in Solid Tumors (RECIST version 1.0) criteria (Eisenhauer et al., 2009). A complete response $(\mathrm{CR})$ was recorded when the tumor had disappeared completely; a partial response (PR) was recorded when the tumor shrank by more than $30 \%$ of the largest diameter; any response was recorded for any degree of response or a decreased size without mention of the tumor dimension; stable disease (SD) was recorded in cases with no sign of recurrent disease within 6 months or change in the tumor size; and progressive disease (PD) was recorded when there was any degree of tumor size increase. PFS was calculated from the administration date of the study drug until PD 


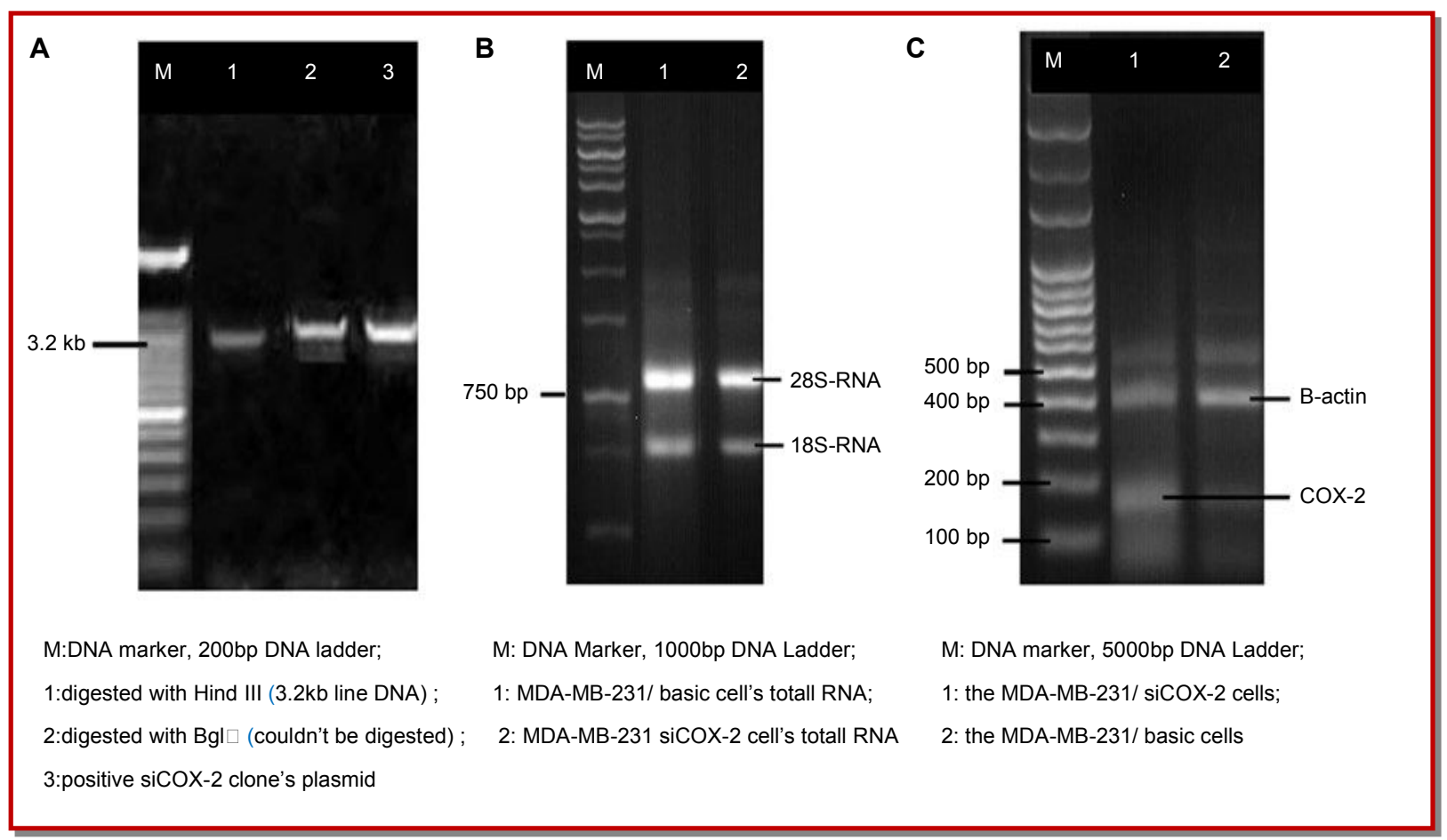

Figure 4: The constructed interferenced plasmid siCOX-2 and levels of COX-2 mRNA. Results of identification of COX-2 with Bgl II and Hind III (A); The detection of cell's totall RNA (B); RT-PCR analysis showed COX-2 mRNA levels of siCOX-2 were obviously reduced at 48 hours after transfection (C)

or death from any cause. Because of the possibility that some patients with no evidence of disease may have artificially influenced the PFS, we checked for the heterogeneity in PFS among patients grouped by their treatment before entering the study (no local treatment vs. surgical or local radiotherapy vs. whole-breast radiotherapy). Among 86 evaluable patients 58 patients achieved SD, 28 patients achieved PD, and 55 patients experienced some degree of radiologic improvement. In all patients, the overall response rate was $37.2 \%$, with a median PFS of 23 months (95\% CI $=11.3-26.0)$ and a median OS of 62 months (95\% CI $=42.7-67.2)$. For COX2 expression, the patients were divided into negative and positive two groups. Considering only the COX-2 expression, survival was found to be longer in negative group than that in positive group, and there were slightly statistically significant between 95\% CI $=1.715$ 2.843 , the two groups $\left(\chi^{2}=9.567, \mathrm{p}=0.0448\right)$. KaplanMeier survival curves demonstrated survival differences between COX-2 positive and COX-2 negative patients $(p=0.0448)$, shown in Figure 3. Other factors which may be associated with COX-2 in breast cancer, which should be investigated in further studies.

In vitro studies by reducing COX-2 by using RNA interference in cells line MDA-MB-231

The results of restriction endonuclease digestion electrophoresis and DNA sequencing showed that the interferenced plasmid siCOX-2 was constructed successfully. The cell strains transfected were named as MDAMB-231/basic and MDA-MB-231/siCOX-2 (Figure
4AB). The levels of COX-2 mRNA detected by RT-PCR shown in Figure 4C. The results of RT-PCR showed that the levels of COX-2 mRNA of MDA-MB-231/siCOX-2 $(0.9 \pm 0.1)$ were obviously reduced at 48 hours after transfection, compared to MDA-MB-231/basic cells (0.3 $\pm 0.1)(\mathrm{p}<0.01)$.

When a scratch was created in the fluent monolayer cells, it took the COX-2-reduced MDA-MB-231 cells a longer time to fill the gap in the scratch assay $(p<0.05)$. Figure 5A showed COX-2-reduced MDA-MB-231 cells showed decreased chemotaxis ability compared with the control cells $(p<0.01)$. The COX-2-reduced MDA-MB -231 cells which were induced by EGF showed decreased chemotaxis and directional migration ability by using RNA interference. Adhesion assay showed in Figure 5B. The number of the adhering cells was decreased for reduction of COX-2 within 5 and 15 minutes $(p<0.01)$. The adhering ability of the COX-2reduced MDA-MB-231 cells was reduced obviously when the cells were adhered in fibronectin. Using RNA interference, the reduction of COX-2 expression can obviously inhibit the invasion and chemotaxis of the highly malignant breast cancer cell line MDA-MB-231 in vitro (Figure 5C). The invasion assay showed prominent differences between the COX-2-reduced MDA-MB-231 cells and the control cells $(\mathrm{p}<0.01)$.

\section{Discussion}

The pathogenesis of breast cancer is not fully clear, 


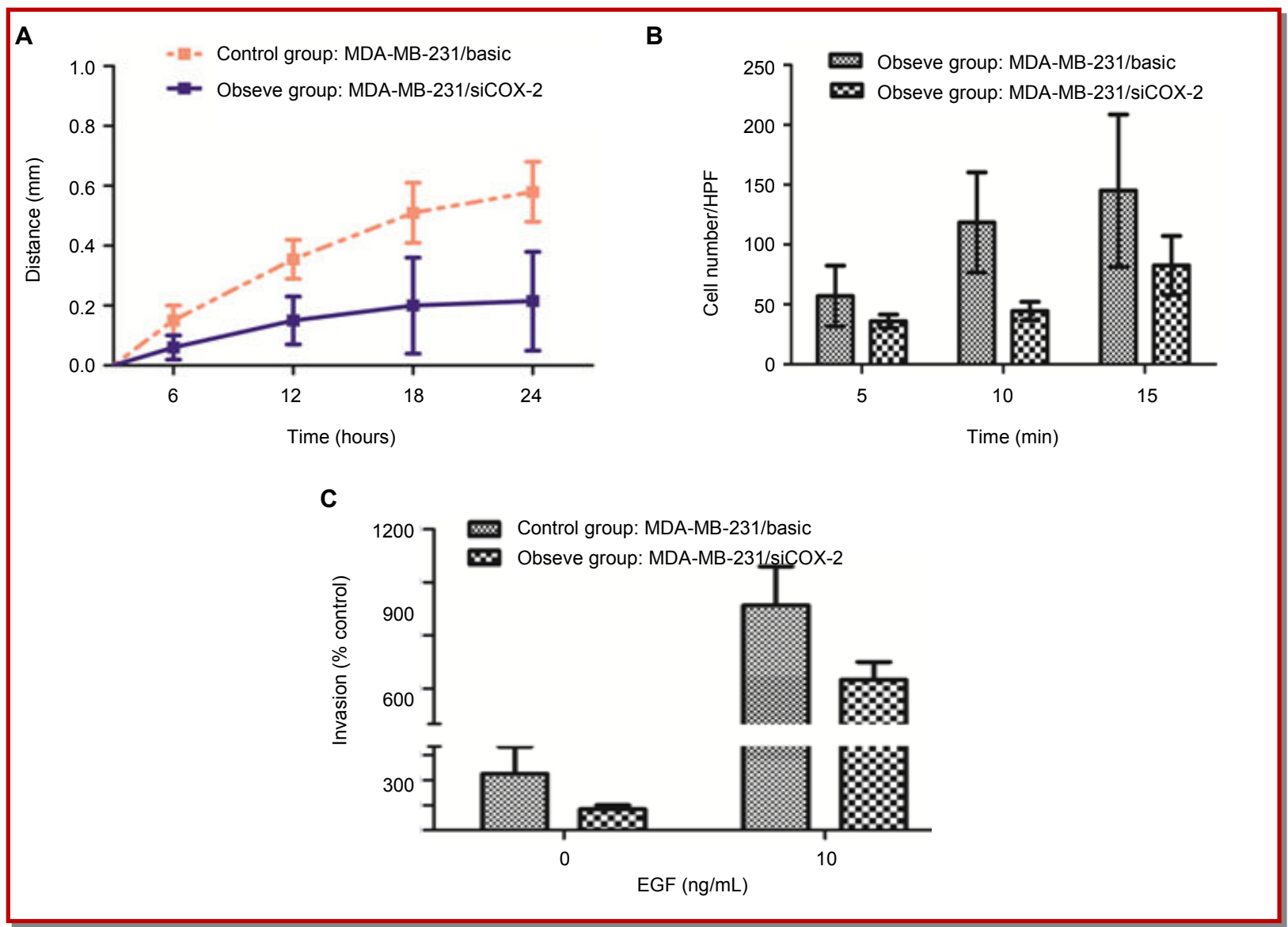

Figure 5: Chemotaxis, adhesion and invasion ability of COX-2 reduced MDA-MB-231 cells by siRNA. COX-2-reduced MDA-MB231 cells showed decreased chemotaxis ability compared with the control cells (A). Adhesion assay showed the number of the adhering cells was decreased for reduction of COX-2 within 5 and $15 \mathrm{~min}$ (B). Comparison of disruption of COX-2 by the siRNA impaired invasion into matrivgel in the MDA-MB-231/basic and MDA-MB-231/ siCOX-2 cells (C)

studies have shown that the proliferation and metastasis of breast cancer cells is a very complex, multistep process, affected by various factors including the common role of environmental, genetic and so on (Sueoka et al., 2013). It is critical for breast cancer management to identify those biomarkers that would provide, at the time of diagnosis, a reliable prognostic assessment and prediction of treatment response (Diers et al., 2013; Cao et al., 2014; Witteveen et al., 2015). Several studies have shown that COX-2 is implicated in carcinogenesis and tumor progression in many human cancers, including breast cancer. However, other studies report there is no significant correlations between COX-2 expression and histopathological parameters or disease-free survival, moreover, roles for COX-2 in invasion and with other molecular biomarkers remain unclear. In the current study, the results show that COX-2 overexpression in breast cancers correlates with poor clinicopathological parameters such as invasion and metastasis in vivo and also obviously inhibit the invasion and chemotaxis by reduction of COX-2 expression of cancer cell line MDAMB-231 using RNA interference in vitro. In addition, This study data demonstrated that the positivity correlated significantly with HIF-1a, VEGF in breast cancer. Therefor, COX-2 overexpression in breast cancer may be considered as a negative prognostic marker.

In the current report, tentatively put forward COX-2 overexpression role on invasivenes, the study was disigned to investigate COX-2 overexpression on invasion and metastasis in human breast cancers tissue, and further invasivenes the influences of disruption of COX-2 express using siRNA plasmids to disrupt COX-2 expression to show RNA interference on invasiveness of highly malignant breast cancer cell line in vitro. In human breast tissue study, its possible COX-2 association with clinicopathological features and prognostic molecular markers such as ER, PR, HER2, HIF-1a, VEGF and MVD was also performed. This results indicate that in human tissues, the COX-2 immune positivity and percentage of positive cells in breast invasive ductal carcinomas was $78.9 \%$ with moderate positive to strong some degree of positivity, in contrast, in benign lesions tissues, COX-2 was weekly expressed only in $16.7 \%$, the expression of COX-2 in cancer tissues was obviously higher than the expression in benign lesions tissues, the difference was statistically signifi- 
cant. These data which can be consistent with the notion that a possible role for COX-2 in carcinogenesis.

In this study, next, the effect of COX-2 expression in breast cancer with clinicopathological features, including histological grade, clinical, lymph node metastasis, distant metastasis and vascular invasion etc., difirrent biological behavior were examined. Results indicate that COX-2 expression immune positivity correlated significantly with HIF-1a, VEGF, the grading, lymph node metastasis, distant metastasis and vascular invasion in breast carcinoma, and shorter overall survival in carcinomas patients group related to COX-2 overexpression in tumors. The finding was consistent with the previous finding that demonstrate that COX-2 overexpression in breast cancers correlates with poor clinicopathological parameters such as invasion and metastasis. On the other hand, to explore the function of reducing COX-2 expression can possible obviously inhibit the invasion, next, set out to performed experiment using siRNA plasmids to disrupt COX-2 expression in vitro role studies. RT-PCR analysis showed COX-2 mRNA levels of siCOX-2 were obviously reduced at 48 hours after transfection; the COX-2reduced MDA-MB-231 cells showed decreased chemotaxis ability $(p<0.01)$; the invasion assay showed prominent differences between the COX-2 reduced MDA-MB231 cells and the control cells $(p<0.01)$. Thus, at current time, the finding was consistent with the previous finding that COX-2 overexpression in breast cancers correlates with poor clinicopathological parameters such as invasion and metastasis; The data demonstrated that the reduction of COX-2 expression can obviously inhibit the invasion and chemotaxis of cancer cell line MDA-MB-231. Taken together, these data indicate that COX-2 overexpression in breast cancer may be considered as a negative prognostic marker. COX-2 is an inducible enzyme that interferes with tumor development and angiogenesis, related to the inhibition of apoptosis through inhibition of the proapoptotic Bax protein and overexpression of the antiapoptotic bcl-2 protein. COX-2 catalyzes the conversion of arachidonic acid to PGE2 and enhances the metastatic phenotype of both breast cancer cells in vitro and breast tumors (Mitchell et al., 2010; Miglietta et al., 2010). PGE2, the catalytic product of COX-2, may promote tumor development and angiogenesis (Boland et al., 2004; Miglietta et al., 2010; Yu et al., 2014). It has been investigated in several human cancers and also correlated with the evolution of the disease (Boland et al., 2004).

Some authors reported that COX-2 up-regulation in a wide variety of malignant solid tumors, including colon, gastric, breast cancer, and its overexpress leads to increased production of prostaglandins that are involved in different physiological and pathophysiological processes, suggesting a possible role for COX-2 in proliferation, carcinogenesis, development, apoptosis, angiogenesis, invasion, and metastasis. In the findings of the present study, we confirm that human breast carcinomas aberrantly express COX-2, and that raised tissue levels of COX-2 may have prognostic value. Patients expressing high levels of COX-2 can develop local recurrence, and have reduced diseasefree and disease-related overall survival. Breast cancer is a heterogeneous disease with multiple genetic alterations able to affect tumor growth, progression and metastasis. However, the pathogenesis of breast cancer is not fully clear, studies have shown that the proliferation and metastasis of breast cancer cells is a very complex, multistep process, affected by various factors including the common role of environmental, genetic and so on (Miglietta et al., 2010). The findings of the present study suggest that the increased expression of COX-2 and HIF-1a, VEGF and MVD-CD105 positively expressive correlation played an important role in the development of breast cancer and might be responsible for the enhanced activity in tumor-induced neovascularization, invasion and metastasis.

We tentatively put forward that increased COX-2, HIF$1 a$, VEGF and MVD may serve as valuable indicators of biological behavior of breast cancer. Hypoxia, is a common feature of various cancers. Solid tumors are characterized by regions of low oxygen tension, which play a central role in tumor progression and resistance to therapy. Cells under hypoxic conditions develop numerous adaptive responses to hypoxic stress concurrently with altered expression of hundreds of genes that are regulated by hypoxia inducible factors (Sueoka et al., 2013; Majmundar et al., 2010). Low oxygen tension affects mitochondrial function and for the cells to survive, mitochondria must functionally adapt to low oxygen tension to maintain the cellular bioenergetics. HIF-1a is an important cellular survival protein under hypoxic conditions, regulating the cellular response to low oxygen tension via recruitment of a transcriptional co-activator, induces expression of multiple genes involved in cell survival, proliferation, angiogenesis, and tumor development (Dewi and Fatchiyah, 2013; Semenza et al., 2010). Angiogenesis is essential for the growth and metastasis of major solid tumors and has been correlated with prognosis in human cancer (Leo et al., 2006). VEGF capable of promoting angiogenesis exerts an important effect in the process of genesis, development, metastasis and recurrence of various tumors. In the process of tumor genesis and development, tumor regenerative capillaries capable of providing nutrients for tumor cells and favorable conditions for distal metastasis are the precondition to induce the local growth, infiltration and distal metastasis of malignant tumors, hence, how to inhibit tumor angiogenesis is a new research hotspot 
at present. VEGF, one of the key factors to promote tumor angiogenesis and with the strongest function and highest specificity, can not only promote the proliferation of endothelial cells, but also regulate and participate in angiogenesis (Kelly et al., 2003). Due to an intimate association with genesis, development, metastasis and infiltration, it is an important indicator to judge the cancer metastasis and infiltration in clinic (Thielemann et al., 2013; Wang et al., 2013; Wang et al., 2014). In the study, VEGF expression in breast carcinoma $(60.9 \%)$ was higher than that in benign tissue and the expression of VEGF was positively correlated with COX-2 in breast carcinoma $(\mathrm{p}<0.05)$. In our study, we also investigated the prognostic significance of CD105 and MVD assessed based on the number of CD105-positive vessel in tissue of breast cancer and epithelium adjacent to the lesion. The MVD in COX-2 positive group was higher than that in COX-2 negitive group, there were slightly correlated with MVD. Tumor angiogenesis and its clinical significance have been studied in a variety of neoplasms. Angiogenesis contributes to the pathogenesis of various cancer, and microvessel density may improve our ability to predict breast cancer extension. Microvessel densities are significantly greater in the primary tumors of patients with metastatic disease than in those without metastases. This conformed that the overexpression COX-2 in breast cancer might be important biological markers for invasion and lymph node metastasis, and the combined detection of COX-2, HIF-1a, VEGF and MVD are benefit to better prewarning markers for monitoring their metastasis in clinic judging the patients with breast carcinoma (Koyama et al., 2010; Dallas et al., 2008; Fonsatti et al., 2010).

Breast cancer is mainly a multifactorial disease, occurring as a result of the combined effects of factors. Many studies have found that certain biological indicators by detecting abnormal expression of HER2/ neu, ER, PR etc., can guide clinical diagnosis and treatment activities prognosis. In addition, several reports have described that in ER - negative patients, but not in ER-positive ones, up-regulation of COX-2 is associated with reduced DFS (Yu et al., 2014). In ERnegative tumors, increased COX-2 expression may contribute to breast cancer progression and the suggested mechanisms include increased prostaglandin production, which, in turn, would promote angiogenesis, and ultimately metastasis (Kim et al., 2014). However, our results in this study suggest that COX-2 immune positivity not significantly correlated with HER2/neu, ER, PR. Moreover, several studies have shown that increased levels of COX-2 are frequently associated with younger patient age, generally, with a more aggressive phenotype (Costa et al., 2002).
Unfortunately, our results suggest that COX-2 immune positivity not significantly correlated with biological parameters such as age, size of tumor, etc. We have to point out that we do not detect every aspect of test cases, and we only detect few biological parameters in the present study. It also should be noted that the number of cases is too limited, and this study has examined only 128 cases infiltrating ductal carcinoma, not included the subtype of breast carcinomas. Other factors which may be associated with COX-2 in breast cancer, which should be investigated in further studies. It is critical for breast cancer management to identify those biomarkers that would provide those factors potentially curable given the availability of early diagnosis and effective therapeutic protocols, at the time of diagnosis, a reliable prognostic assessment and prediction of treatment response (Thorat et al., 2013; Peng et al., 2013 ).

In this study, the results revealed that patients with increased COX-2 expression have shorter survival times. A slightly correlation between COX-2 expression and prognostic factors in breast cancer suggesting that increased COX-2 expression was related to worse prognosis. The result suggests that the difference between COX-2 expression in the breast cancer may related to the differentiated behavior of these tumors, confirming the association between the COX-2 expression and disease aggressiveness. This study showed shorter survival in patients whose tumors expressed more COX-2. Therefore, there are possibility of using COX-2 inhibitor anti-inflammatory drugs to treat mammary tumors. When overall survival was assessed, the results suggesting that COX-2 is essential for the development and evolution of neoplastic disease, resulting in shorter overall survival and worse prognosis. This study suggests that the increased expression of COX-2 in tumors indicates a higher risk of development and dissemination of the disease.

\section{Conclusion}

COX-2 overexpression correlates with poor clinicopathological parameters such as invasion and metastasis in breast cancers, and the reduction of COX2 expression can obviously inhibit the invasion and chemotaxis of cancer cell line MDA-MB-231 using RNA interference. The findings of the present study suggest that COX-2 overexpression in breast cancer may be considered as a negative prognostic marker.

\section{Ethical Issue}

The study protocol was endorsed by the Medical Ethical Committee of the Hospital; besides, each participant provided informed consent. 


\section{Conflict of Interest}

The author confirms that this article content has no conflict of interest.

\section{Acknowledgement}

This work was financially supported by Project of Application Technology Research and Development Project Foundation in Rizhao (2014SZSH02), Medicine and Health Care Science and Technology Development Plan Project Foundation of Shandong Province (2014WS0282 and 2014WSA11003), Medical Staff' Science and Technology Innovation Projects of Shandong Province Medical Union Committee (201515), Scientific Research Key Projects of Jining Medical University (JY2013KJ051), as well as Students' Innovative Training Program of Jining Medical University (CX2015079). We also thanks to Prof. Wen-Jun Guo and Dr. Ping-Ping Zhao (Pathology Department of Medical College, Weifang Medical University) for their valuable work for this study.

\section{References}

Aggarwal A, Al-Rohil RN, Batra A, Feustel PJ, Jones DM, DiPersio CM. Expression of integrin $\alpha 3 \beta 1$ and cyclooxygenase-2 (COX2) are positively correlated in human breast cancer. BMC Cancer. 2014; 14: 459.

Boland GP, Butt IS, Prasad R, Knox WF, Bundred NJ. COX-2 expression is associated with an aggressive phenotype in ductal carcinoma in situ. Br J Cancer. 2004; 90: 423-29

Cao J, Yang X, Li WT, Zhao CL, Lv SJ. Silencing of COX-2 by RNAi modulates epithelial-mesenchymal transition in breast cancer cells partially dependent on the PGE2 cascade. Asian Pac J Cancer Prev. 2014; 15: 9967-72.

Costa C, Soares R, Reis-Filho JS, Leitão D, Amendoeira I, Schmitt FC. Cyclo-oxygenase 2 expression is associated with angiogenesis and lymph node metastasis in human breast cancer. J Clin Pathol. 2002; 55: 429-34.

Dallas NA, Samuel S, Xia L, Fan F, Gray MJ, Lim SJ, Ellis LM. Endoglin (CD105): A marker of tumor vasculature and potential target for therapy. Clin Cancer Res. 2008; 14: 193137.

Dewi FR, Fatchiyah F. Methylation impact analysis of erythropoietin (EPO) Gene to hypoxia inducible factor-1a (HIF-1a) activity. Bioinformation 2013; 9: 782-87.

Diers AR, Vayalil PK, Oliva CR, Griguer CE, Darley-Usmar V, Hurst DR, Welch DR, Landar A. Mitochondrial bioenergetics of metastatic breast cancer cells in response to dynamic changes in oxygen tension: Effects of HIF-1a. PLoS ONE. 2013; 8: e68348.

Eisenhauer EA, Therasse P, Bogaerts J, Schwartz LH, Sargent D, Ford R, Dancey J, Arbuck S, Gwyther S, Mooney M, Rubinstein L, Shankar L, Dodd L, Kaplan R, Lacombe D, Verweij J. New response evaluation criteria in solid tumours: Revised RECIST guideline (version 1.1). Eur J Cancer. 2009; 45: 228-47.

Elston CW, Ellis IO. Pathological prognostic factors in breast cancer. I. The value of histological grades in breast cancer:
Experience from a large study with long-term follow-up. Histopathology 1991; 19: 403-10.

Fonsatti E, Nicolay HJ, Altomonte M, Covre A, Maio M.Targeting cancer vasculature via endoglin/CD105: A novel anti-body-based diagnostic and therapeutic strategy in solid tumours. Cardiovasc Res. 2010, 86: 12-19.

Kelly LM, Hill AD, Kennedy S, et al. Lack of prognostic effect of Cox-2 expression in primary breast cancer on short-term follow-up. Eur J Surg Oncol. 2003; 29: 707-10

Khan S, Ferguson B H, Asuncion V MM, Turay D, Diaz Osterman CJ, Moyron RB, Esebanmen GE, Ashok A, Wall NR. Localization and up-regulation of surviving in cancer health disparities: A clinical perspective. Biol Targets Ther. 2015; 9: 57-67.

Kim MJ, Kim HS, Lee SH, Yang Y, Lee MS, Lim JS. NDRG2 controls COX-2/PGE2-mediated breast cancer cell migration and invasion. Mol Cells. 2014; 37: 759-65.

Kopczyńska E, Makarewicz R. Endoglin: A marker of vascular endothelial cell proliferation in cancer. Wspolczesna Onkol. 2012; 16: 68-71.

Koyama Y, Okayama H, Kumamoto K, Saito K, Nakamura I, Ohki S, Takenoshita S. Overexpression of endoglin (CD105) is associated with recurrence in radically resected gastric cancer. Exp Ther Med. 2010; 1: 627-33.

Lakhani SR, Ellis IO, Schnitt SJ, Tan PH, Vijver MJVD, (eds). WHO classification of tumours of the breast. $4^{\text {th }}$ ed. France, IARC Press, 2012, pp 1-117.

Leo C, Faber S, Hentschel B, Höckel M, Horn LC. The status of cyclo-oxygenase-2 expression in ductal carcinoma in situ lesions and invasive breast cancer correlates to cyclooxygenase-2 expression in normal breast tissue. Ann Diagn Pathol. 2006; 10: 327-32

Majmundar AJ, Wong WJ, Simon MC. Hypoxia inducible factors and the response to hypoxic stress. Mol Cell. 2010; 40: 294-309.

Miglietta A, Toselli M, Ravarino N, Vencia W, Chiecchio A, Bozzo F, Motta M, Torchio B, Bocca C. COX-2 expression in human breast carcinomas: Correlation with clinicopathological features and prognostic molecular markers. Expert Opin Ther Targets. 2010; 14: 655-64.

Mitchell K, Svenson KB, Longmate WM, Gkirtzimanaki K, Sadej R, Wang X, Zhao J, Eliopoulos AG, Berditchevski F, Dipersio CM. Suppression of integrin a $3 \beta 1$ in breast cancer cells reduces cyclooxygenase-2 gene expression and inhibits tumorigenesis, invasion, and cross-talk to endothelial cells. Cancer Res. 2010; 70: 6359-67.

Olivan M, Rigau M, Colás E, Garcia M, Montes M, Sequeiros T, Regis L, Celma A, Planas J, Placer J, Reventós J, de Torres I, Doll A, Morote J. Simultaneous treatment with statins and aspirin reduces the risk of prostate cancer detection and tumorigenic properties in prostate cancer cell lines. BioMed Res Int. 2015; 2015: 762178.

Peng L, Zhou Y, Wang Y, Mou H, Zhao Q. Prognostic significance of COX-2 immunohistochemical expression in colorectal cancer: A meta-analysis of the literature. PLoS One. 2013; 8: e58891. 
Ranger GS, Jewell A, Thomas V, Mokbel K. Elevated expression of cyclooxygenase-2 in breast cancer and ductal carcinoma in situ has no correlation with established prognostic markers. J Surg Oncol. 2004; 88: 100-03.

Semenza GL. Defining the role of hypoxia inducible factor 1 in cancer biology and therapeutics. Oncogene 2010; 29: 625-34.

Sueoka E, Sueoka-Aragane N, Sato A, Ide M, Nakamura H, Sotomaru Y, Taya C, Yonekawa H, Kitagawa T, Kubota Y, Kimura S, Nakachi K, Tanimoto K. Development of lymphoproliferative diseases by hypoxia inducible factor-1 a is associated with prolonged lymphocyte survival. PLoS One. 2013; 8: e57833.

Thielemann A, Baszczuk A, Kopczyński Z, Kopczyński P, Grodecka-Gazdecka S. Clinical usefulness of assessing VEGF and soluble receptors sVEGFR-1 and sVEGFR-2 in women with breast cancer. Ann Agric Environ Med. 2013, 20: 293-97.

Thorat D, Sahu A, Behera R, Lohite K, Deshmukh S, Mane A, Karnik S, Doke S, Kundu GC. Association of osteopontin and cyclooxygenase-2 expression with breast cancer subtypes and their use as potential biomarkers. Oncol Lett. 2013; 6: 1559-64.

Wang G, Qin Y, Zhang J, Zhao J, Liang Y, Zhang Z, Qin M, Sun Y. Nipple discharge of CA15-3, CA125, CEA and TSGF as a new biomarker panel for breast cancer. Int J Mol Sci. 2014;
15: 9546-65.

Wang GP, Qin MH, Liang YA, Zhang $H$, Zhang ZF. The significance of biomarkers in nipple discharge and serum in diagnosis of breast cancer. In: Advanced engineering and technology. Xie L, Huang D (eds.). CRC Press, 2014, pp 66571 .

Wang JJ, Sun XC, Hu L, Liu ZF, Yu HP, Li H, Wang SY, Wang DH. Endoglin (CD105) expression on microvessel endothelial cells in juvenile nasopharyngeal angiofibroma: Tissue microarray analysis and association with prognostic significance. Head Neck. 2013; 35: 1719-25.

Witteveen A, Kwast AB, Sonke GS, IJzerman MJ, Siesling S Survival after locoregional recurrence or second primary breast cancer: Impact of the disease-free interval. PLoS One. 2015; 10: e0120832.

Yi C, Zhang Y, Yu Z, Xiao Y, Wang J, Wang J, Qiu H, Yu W Tang R, Yuan Y, Guo W, Deng W. Melatonin enhances the anti-tumor effect of fisetin by inhibiting COX-2/iNOS and NF-kB/p300 signaling pathways. PLoS ONE. 2014; 9: e99943.

Yu KR, Lee JY, Kim HS, Hong IS, Choi SW, Seo Y, Kang I, Kim JJ, Lee BC, Lee S, Kurtz A, Seo KW, Kang KS. A p38 MAPKmediated alteration of COX-2/PGE2 regulates immunomodulatory properties in human mesenchymal stem cell aging. PLoS ONE. 2014; 9: e102426.

\footnotetext{
Author Info

Gang-Ping Wang (Principal contact)

' e-mail: wgprzph93@126.com; Tel: +86-633-3365056
} 


\section{Your feedback about this paper}

1. Number of times you have read this paper 0

2. Quality of paper
Excellent
Good
Moderate
Not good

3. Your comments

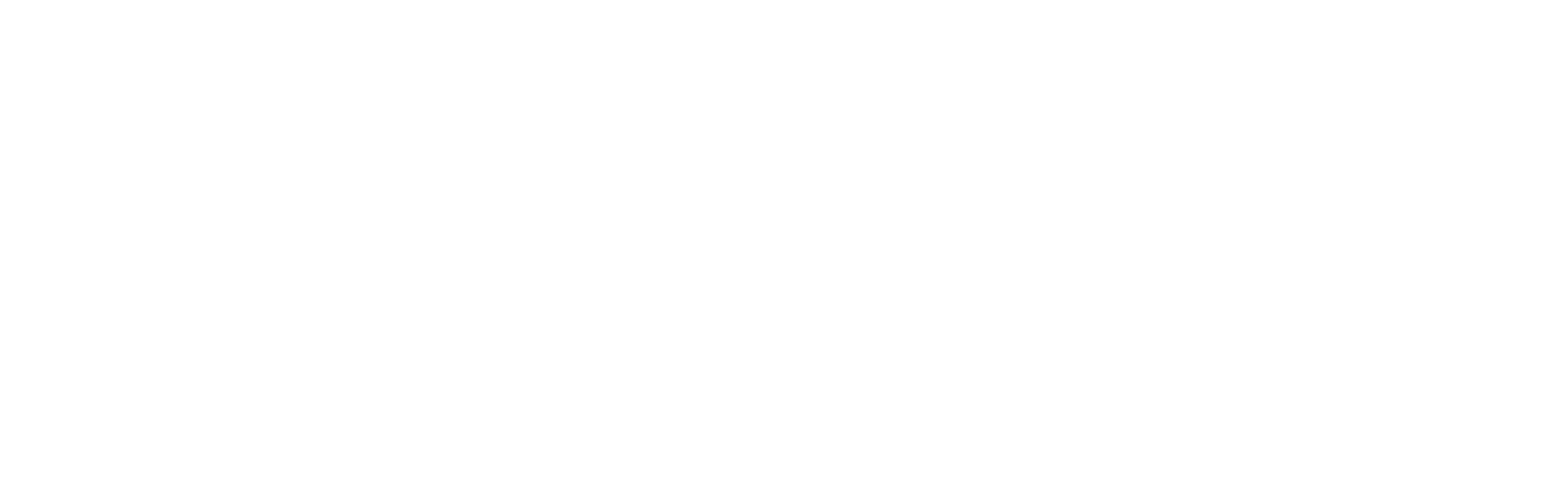

